\title{
Trennung von Harnstoff-Herbiziden und Chloranilinen mittels Hochdruck-Flüssigchromatographie
}

\section{J. Pribyl}

Institut für Wasser-, Boden- und Lufthygiene des Bundesgesundheitsamtes, Postfach, D-1000 Berlin 33, Bundesrepublik

Deutschland

\section{Summary}

- Separation of Urea Herbicides and Chloranilines by HPLC

The LC separation of urea herbicides and chloranilines is described. The latter are found variously as metabolites of the urea compounds.

\section{Zusammenfassung}

Es wird die LC-Trennung von herbiziden Harnstoffen und Chloranilinen beschrieben. Die letzteren treten verschiedentlich als Abbauprodukte der Harnstoffe auf. sich dabei Kieselgel als stationäre Phase und - je nach Art der zu trennenden Substanzgruppen - verschiedene Hexan/Methylenchlorid/Äthanol-Gemische als mobile Phase (isocratisch) erwiesen. Kommen Harnstoff-Herbizide zusammen mit den hinsichtlich der Polarität stark unterschiedlichen Chloranilinen vor, so versagt die hier verwendete isocratische Elution meist, obwohl sie sonst unbestrittene Vorzüge besitzt. Um das Substanzengemisch zu trennen, ist es daher notwendig, die Polarität der mobilen Phase während des chromatographischen Prozesses den zu eluierenden Substanzen anzupassen.

Fig. 1 gibt den hierzu beschrittenen Weg wieder.
Von den einzelnen Gruppen der Herbizide spielen die Phenylharnstoffe eine bedeutende Rolle. Sie treten oftmals zusammen mit verschiedenen Chloranilinen auf, und zwar sowohl bei ihrer Herstellung [1] als auch nach ihrer Anwendung als Pflanzenschutzmittel [2]

Harnstoffe und Aniline sind meist nicht durch Chromatographie in der Gasphase analytisch bestimmbar, können jedoch mit Hilfe der Hochdruck-Flüssigchromatographie getrennt und quantifiziert werden. Einige flüssigchromatographische Parameter der Harnstoffherbizide [3] und der Chloraniline [4] wurden bereits beschrieben. Als günstigstes Trennsystem für Substanzen beider Klassen haben

\section{Literatur}

[1] W. Woerst, Ullmanns Enzyklopädie der technischen Chemie, Band 15, Urban \& Schwarzenberg-Verlag, München-Berlin, 1964, S. 152-153 (Úbersicht).

[2] F. A. Gunther, Residue Reviews 58, 75-89 (1975) (Ubcrsicht).

[3] J. Pribyl, F. Herzel, J. Chromatog. 125, 487-494 (1976).

[4] J. Pribyl, F. Herzel, Z. Anal, Chem. 283, 343-347 (1977). 